\title{
Implicaciones del Deporte Federado hacia la Empatía y Actitud a la Educación Física en Adolescentes \\ Implications of Federated Sports towards Empathy and Attitude to Physical Education in Adolescents \\ *Victor Gómez Sánchez, *Rosario Padial Ruz, *Manuel Gentil Adarve, **Javier Chacón Zagalaz, *Felix Zurita Ortega *Universidad de Granada (España), **Universidad de Jaén (España)
}

\begin{abstract}
Resumen. El estudio evalúa el grado de empatía, tanto a nivel cognitivo como afectivo, y la actitud hacia la educación física, del alumnado de tercer ciclo de Primaria y primer ciclo de Enseñanza Secundaria Obligatoria (ESO) de Andalucía, que participan en deportes federados. Para ello, se realizaron estudios descriptivos y comparativos con las variables empatía cognitiva y afectiva, y actitud hacia la Educación Física, en una muestra de 849 alumnos federados. Los instrumentos utilizados para la obtención de datos fueron el cuestionario de Actitud hacia la Educación Física (CAEF) y Test de Empatía Cognitiva y Afectiva (TECA). Los resultados muestran que los sujetos que tienen una mayor empatía muestran un aumento significativo en la actitud hacia la Educación Física, y más concretamente en la dificultad sobre la misma, la empatía con el profesor y la asignatura, la concordancia con la organización de la asignatura y la preferencia por la Educación Física y el Deporte. Existen diferencias de género en la empatía, tanto a nivel cognitivo como afectivo, siendo éste mayor en las mujeres. Palabras clave: Empatía, Actitud hacia la Educación Física, Escolares Federados.
\end{abstract}

\begin{abstract}
The study assesses the degree of empathy, both cognitive and affective, and the attitude towards physical education, in students from the third cycle of primary and the first cycle of Secondary Compulsory Education (ESO) in Andalusia who participate in federated sports. Descriptive and comparative analyses were carried out on a sample of 849 federated students, the included variables being cognitive and affective empathy, and attitude towards Physical Education. The instruments used to obtain the data were the Action for Physical Education questionnaire (CAEF), and the Cognitive and Affective Empathy Test (TECA). The findings show that subjects with greater empathy display significantly higher attitude towards Physical Education, more concretely towards its difficulty, empathy with teacher and subject, agreement with subject management, and preference for Physical Education and Sports. Gender differences were found for empathy, both cognitive and affective, this being higher in women.
\end{abstract}

Keywords: Empathy, Attitude towards Physical Education, Federated Schoolchildren.

\section{Introducción}

Tras la búsqueda realizada en la literatura científica para la elaboración de este trabajo, podemos destacar que los estudios recientes que se relacionan con la actitud hacia la educación física, reflejan una disminución de la actitud hacia la educación física conforme aumenta la edad, siendo un momento de crisis el paso de la Educación Primaria a la Educación Secundaria, valorando la asignatura de Educación Física de forma más negativa (Aires et al, 2017; Corbin, Pangrazi y LeMasurier, 2004; Moreno, Muñoz, Pérez y Sánchez, 2004; Méndez-Giménez, Martínez de Ojeda y Valverde-Pérez, 2016; Telama, Naul, Nupponen, Rychtecky y Vuolle, 2002). Para dicha búsqueda se introdujeron los parámetros «actitud educación física» tanto en español como en inglés «physical education attitude», en la base de datos Web of Science (WOS), encontrando un número reducido de artículos publicados los últimos cinco años, de los que solo 1 está en español y 151 en inglés. Los estudios reflejan una disminución de la actitud de la Educación Física debido a la edad y a la utilización de la competición.

Un factor importante a tener en cuenta a la hora de motivar al alumnado en su participación en los contenidos relacionados con el deporte en la escuela, es el modelo de competición. Solo un $4 \%$ de la muestra indica como motivante la competición a la hora de realizar deporte. Esta motivación por ganar, está relacionada con el considerable porcentaje de abandono en la práctica deportiva de adolescentes, pues-

Fecha recepción: 18-03-19. Fecha de aceptación: 05-05-19

Victor Gómez Sánchez

vitorgranada@hotmail.com to que produce decepción al no satisfacer las expectativas de rendimiento. De ahí que en otras actividades en las que no priman directamente el perfeccionamiento y la victoria, tengan mayor cuota de participación (García, 1997), siendo el motivo principal para la realización de actividad física el mantener una buena salud, aunque también existen otras motivaciones como la diversión, relaciones sociales, la imagen corporal, la evasión y por último la competición (Nuviala, Ruiz y García, 2003). Desde el punto de vista de la salud, existen un consenso entre las autoridades sanitarias, al considerar la práctica de actividad física un factor muy importante para tener una buena salud (Zurita et al., 2009), debido a los numerosos beneficios que aporta a nivel físico, psíquico y social (Beltrán, Devís y Peiró, 2012; Guillamón, García y Pérez, 2018; Ortiz y Gómez, 2017; Reloba, Chirosa y Reigal, 2016; Reloba, et al., 2017; Warburton, Nicol y Bredin, 2006). A nivel físico disminuyendo la probabilidad de patologías de origen cardiaco, respiratorio y metabólico entre otras (Hills, King y Amstrong, 2007). A nivel psíquico, proporcionando una imagen corporal más positiva, y reduciendo los niveles de ansiedad y estrés (Strong et al., 2005), y a nivel social, ya que la realización de actividades físicas proporciona valores como la cooperación, amistad, respeto y expresión de sentimientos (Chacón, et al., 2017). Por todo ello, existe relación a nivel profesional entre los implicados en la sanidad y en lo educativo, llevando a cabo investigaciones cuyo objetivo es dotar a las personas de una mejora en su calidad de vida (Bouchard, Shephard, Stephens, Sutton y McPherson, 1990; Ministerio de Sanidad, 2012; Ramírez, Vinaccia y Suárez, 2004; Serra, Zaragoza y Generelo, 2014).

La actividad física proporciona salud y calidad de vida (Camacho-Miñano, García, Rico y Ángel, 2013), y dentro de 
esta se encuentra el deporte como una práctica ligada a ella. Existiendo dos tipos diferentes de deporte, el escolar y el de competición (Cruz, Boixadós, Torregrosa y Mimbrero, 1996; González, Manrique y López, 2012; López, Prieto, Samalot y Madrona, 2016; Manrique, López, Monjas, Barba y Gea, 2011; Usán, Salavera, Merino y Jarie, 2018). En el ámbito escolar, la educación física tiene como uno de sus principales contenidos el deporte en la escuela (González y Pastor, 2005). El área de Educación Física, dentro de su currículum escolar, contiene el deporte como uno de sus elementos motivadores para la consecución de habilidades, destrezas motrices, desarrollo personal y calidad de vida, donde prima la formación, la integración y la mejora de valores personales (Kane, 2015; Navarro-Patón, Rodríguez y Eirín, 2016). Por otro lado, el deporte competitivo proporciona a sus participantes valores instrumentales como trabajo en equipo, cooperación, liderazgo, disciplina, compromiso, afán de superación, fuerza de voluntad, logro, éxito, autocontrol de los impulsos, salud, automotivación, persistencia y valores finales o éticos. Estos últimos, divididos a la vez en valores de identificación emocional (respeto, empatía, compasión, humanidad, indulgencia y solidaridad), y de sacrificio propio (honradez, honestidad, integridad, abnegación, altruismo, generosidad y sacrificio) (Durán, 2013; López, Pérez, Manrique y Monjas, 2016; Ruiz, Ponce, Sanz y Valdemoros, 2015).Todos estos valores, que proporciona el deporte competición,se enmarcan fuera del ámbito educativo, siendo las diferentes federaciones las que regulan su desarrollo (López y Castejón, 2005; Reverter, Mayolas y Adell, 2009; Romero, 2004). Estas entidades recogen y controlan los diferentes clubes que integran los deportes. Sus fines están bien delimitados y persiguen como objetivo principal el resultado (Rojas, 1994).

La realización de un deporte competitivo, implica una serie de valores y competencias emocionales (Muñoz, Ramos y Benítez, 2013) dentro de los cuales encontramos la empatía, que según Salovey y Mayer (1990), queda recogida en uno de los cinco dominios principales de la Inteligencia Emocional, en concreto en el de reconocer las emociones de los demás, donde la empatía hace que seamos capaces de sintonizar con los deseos y necesidades de los demás. Goleman (2011), basándose en la teoría anterior, afirma que la inteligencia emocional es un conjunto de habilidades con cinco componentes: el autoconocimiento, la autorregulación, la automotivación, las habilidades sociales y la empatía, esta última se conceptualiza como una respuesta afectiva donde las personas son capaces de comprender el estado emocional de otra persona sabiendo lo que sentirán en diferentes situaciones o contextos sociales (Eisenberg y Fabes,1998). Este proceso psicológico es diferente en la personas, lo que hace que los sujetos seamos diferentes a la hora de desarrollar este proceso mental (Farrington, Jolliffe, Loeber, Stouthame y Kalb 2001), y por tanto influye directamente en el comportamiento de los sujetos en las diferentes situaciones que se nos dan (Eisenberg et al., 1999; Kaukiainen et al., 1999). En la actualidad, la baja empatía emocional se relaciona directamente con las conductas violentas y antisociales de los sujetos, no siendo capaces de ser aprehensivos ni comprender el estado emocional del otro sujeto, lo que lleva a actuar de forma agresiva en contextos sociales, sobre todo en el contexto escolar (Anderson y Bushman, 2002; Evans, Heriot y
Friedman, 2002; Hoffman, 2000; Stormont, 2002).

Partiendo de la práctica deportiva federada a nivel competitivo en escolares (Gutiérrez, Tomás y Calatayud, 2018), y teniendo en cuenta que la competición puede desarrollar la empatía y la actitud del alumnado hacia la Educación Física, este estudio tiene como objetivo analizar el grado de empatía y actitud hacia la Educación Física del alumnado de tercer ciclo de Primaria y primer ciclo de Enseñanza Secundaria Obligatoria (ESO) de Andalucía que realizan deporte federado y establecer relaciones entre ambas variables.

\section{Materiales y Métodos}

\section{Diseño y participantes}

Se realizó un estudio de tipo descriptivo y corte transversal, en el que la actitud hacia la actividad física, la empatía cognitiva y afectiva se constituyeron como variables dependientes y el sexo y estar federado/a en algún deporte como dependientes, configurando un estudio unifactorial multivariado. La muestra de los centros fue seleccionada de modo aleatorio, obteniendo un total de 849 alumnos/as con una edad comprendida entre 10 y 14 años, donde 316 eran mujeres y 533 hombres. Como criterio de selección de los participantes se determinó que cursaran el tercer ciclo de primaria y primer ciclo de la ESO en la comunidad autónoma de Andalucía, y que estuvieran federados en algún deporte.

\section{Variables}

Las variables usadas en este estudio fueron las siguientes:

- Género: según era masculino o femenino.

- Curso Académico: se eligieron cuatro cursos (quinto y sexto de Primaria y primero y segundo de Educación Secundaria Obligatoria).

• Población de la Comunidad Autónoma Andaluza: establecidos en las ocho provincias andaluzas (Jaén, Córdoba, Sevilla, Huelva, Cádiz, Málaga, Granada y Almería), y sus diferentes municipios.

- Federado: realiza deporte federado colectivo o individual, o solo deporte sin federar.

- Actitud hacia la Educación Física: determinado por siete categorías: valoración de la asignatura y del profesor/a de educación física, dificultad de la educación física, utilidad de la asignatura de educación física, empatía con el profesor/a y la asignatura, concordancia con la organización de la asignatura, la educación física como deporte y preferencia por la educación física y el deporte.

- Empatía: recogiendo dentro de esta la empatía cognitiva, donde se comprende lo que se siente y la empatía afectiva donde sentimos lo que otros/as sienten.

\section{Instrumentos}

Para el desarrollo de este trabajo de investigación se utilizaron tres instrumentos de recogida de datos:

- El primero de ellos, un cuestionario de tipo sociodemográfico donde se determina el sexo (masculino o femenino), edad y practica de actividad física.

- El segundo cuestionario administrado fue el Elaborado por Moreno et al. (1996) de Actitud hacia la Educación Física (CAEF) . Se pregunta a los y las estudiantes el grado 
de conformidad con los siguientes siete factores hipotéticos: evaluación de los aspectos más importantes del profesorado y la asignatura de Educación Física (EF), facilidad de la asignatura y su comparación con otras asignaturas, validez de la asignatura y sus contenidos para la formación integral del alumnado, preocupación del profesorado de Educación Física hacia el alumnado, funcionamiento de la asignatura de Educación física, actitud hacia la Educación Física y el Deporte y comparación entre Educación Física y Deporte. Está compuesto por 56 items destinados a valorar los intereses y actitudes del alumnado de EF, disponiendo de cuatro alternativas de respuesta, desde 1 (en desacuerdo) hasta 4 (totalmente de acuerdo). Valoración de la asignatura y del profesor/a de Educación Física: 6, 10, 17, 18, 21, 30, 37, 38, 43, 51, 55. Dificultad de la Educación Física: 4(-), 11(-), 19(-), 26(), 36(-), 44(-). Utilidad de la Educación Física: 1, 5, 12, 15, 16, 31, 32, 35, 40, 42. Empatía con el profesor y la asignatura: 2, 3, 7, 8, 23, 48. Concordancia con la organización de la asignatura: 13, 14, 24, 52, 54. Preferencia por la Educación Física y el Deporte: 20, 28, 34, 50. La Educación Física como Deporte: 25, 45, 47, 49.

- El tercer instrumento que se empleó fue el Test de Empatía Cognitiva y Afectiva (TECA), elaborado por Gorostiaga, Balluerka y Soroa (2014) que proporciona información tanto de los componentes cognitivos de la empatía (Adopción de perspectivas y comprensión emocional) como de los afectivos (Estrés empático y alegría empática). Además ofrece una puntuación total de la empatía. Permite predecir si una persona va a mostrar empatía en una situación emocional completa. Está formado por 33 ítems. La puntuación total se obtiene sumando las puntuaciones según escala Likert de 5 puntos que indica desde: $1=$ Totalmente en desacuerdo y $5=$ Totalmente de acuerdo. Consta de cuatro factores que forman parte de dos dimensiones: a) DIMENSIÓN COGNITIVA (DC)dividida en dos Subescalas: Adopción de perspectivas (AP) y comprensión emocional (CE); y b) DIMENSIÓN EMOCIONAL (DE) con otras dos Subescalas: estrés empático (EE) y alegría empática (AE).

\section{Procedimiento}

Los cuestionarios se administraron en horario de clase y se aplicaron de manera grupal por los/as investigadores, preparados para tal efecto, después de hablar con los/as propios/as estudiantes y tras presentar el consentimiento informado por parte de los/as representantes legales. Se garantizó a los/as estudiantes el anonimato de la información recogida, aclarando que su utilización sería sólo con fines científicos.

\section{Análisis de los Datos}

El análisis estadístico se realizó a través del software IBM SPSS ${ }^{\circledR}$ 24.0. Para los descriptivos básicos se emplearon frecuencias y medias, mientras que para el estudio de relaciones entre variables se ha utilizado t de Student y Anova de un factor dependiendo de las categorías de las variables. La fiabilidad interna de los instrumentos empleados fue valorada mediante el á de Cronbach, fijando el índice de confiabilidad en el 95.5\%.

\section{Resultados}

En cuanto al estudio descriptivo de este trabajo de investigación en primer lugar los resultados depararon que la muestra estaba formada por 316 mujeres y 533 hombres.

En los niveles de empatía, la dimensión cognitiva estaba incrementada ( $\mathrm{M}=3.41$ ) respecto a la afectiva $(\mathrm{M}=3.24)$. En lo que respecta a la actitud hacia la asignatura de Educación Física, el ítem de concordancia con la organización de la asignatura es el más valorado ( $M=3.06)$, mientras que la utilidad que tiene es la menos valorada ( $\mathrm{M}=2.07)$, como se ob-

Tabla 1

\begin{tabular}{|c|c|c|c|c|c|c|c|}
\hline & & $\mathrm{N}$ & $\%$ & & & $\mathrm{M}$ & DT \\
\hline \multirow[t]{2}{*}{ Sexo } & Hombres & 533 & 62.8 & \multirow{5}{*}{$\begin{array}{l}\text { Actitudhaci } \\
\text { a la EF }\end{array}$} & $\begin{array}{l}\text { Valoración de la asignatura } \\
\text { y del profesor de EF }\end{array}$ & 2.77 & 0.513 \\
\hline & Mujeres & 316 & 37.2 & & Dificultad de la EF & 2.47 & 0.557 \\
\hline \multirow{4}{*}{ Empatía } & & $\mathrm{M}$ & DT & & Utilidad de la EF & 2.07 & 0.445 \\
\hline & \multirow[b]{2}{*}{$\begin{array}{l}\text { Dimensión } \\
\text { Cognitiva }\end{array}$} & \multirow[b]{2}{*}{3.41} & \multirow[b]{2}{*}{0.430} & & $\begin{array}{c}\text { Empatía con el profesor y } \\
\text { la asignatura }\end{array}$ & 2.52 & 0.585 \\
\hline & & & & & $\begin{array}{c}\text { Concordancia con la } \\
\text { organización de la } \\
\text { asignatura }\end{array}$ & 3.06 & 0.594 \\
\hline & $\begin{array}{l}\text { Dimensión } \\
\text { Afectiva }\end{array}$ & 3.24 & 0.420 & & $\begin{array}{c}\text { Preferencia por la EF y el } \\
\text { Deporte }\end{array}$ & 2.42 & 0.608 \\
\hline
\end{tabular}

Tabla 2.

\begin{tabular}{|c|c|c|c|c|c|}
\hline \multicolumn{2}{|c|}{ Relación entre la empatia y el s } & Media & D.T & \multirow{3}{*}{$\begin{array}{c}\mathrm{F} \\
4.978\end{array}$} & \multirow{3}{*}{$\begin{array}{c}\mathrm{p} \\
0.026\end{array}$} \\
\hline Dimensión & Masculino & 3,38 & 0.425 & & \\
\hline Cognitiva & Femenino & 3,45 & 0.436 & & \\
\hline Dimensión & Masculino & 3,23 & 0.428 & & \\
\hline Afectiva & Femenino & 3,26 & 0.406 & 0.699 & 0.403 \\
\hline
\end{tabular}

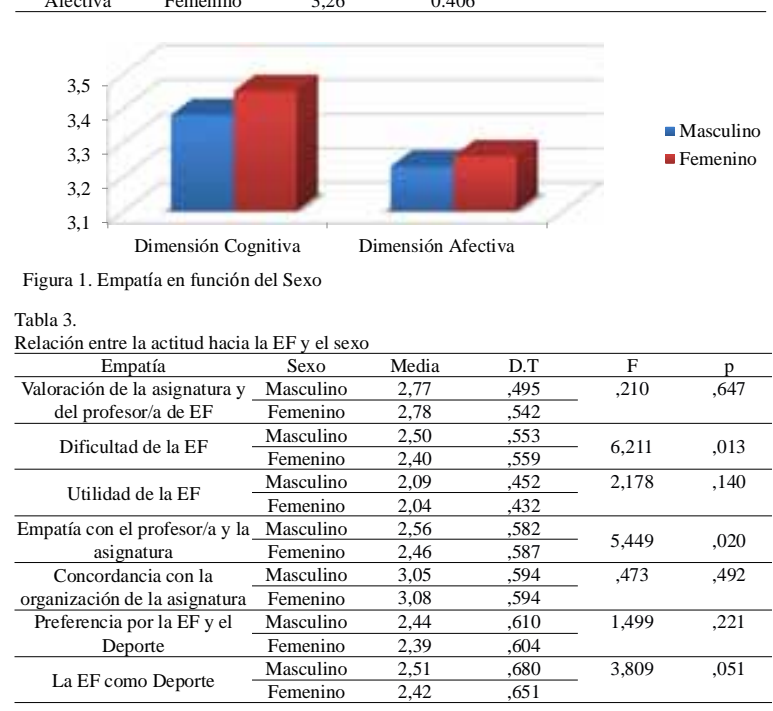

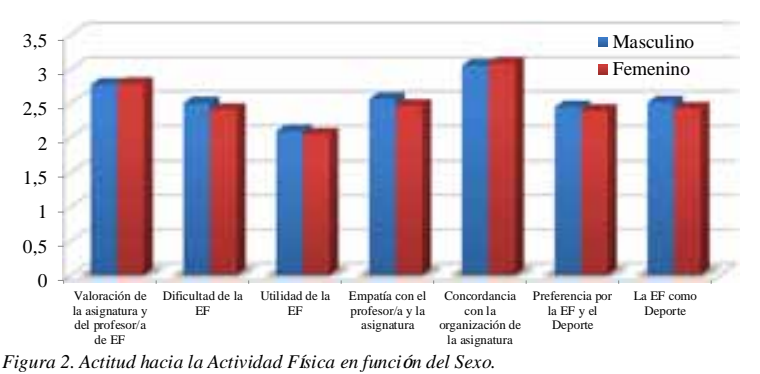

Figura 2. Actitud hacia la Actividad Física en función del Sexo.

Tabla 4

Relación entre empatía y la actitud hacia la EF

\begin{tabular}{|c|c|c|c|c|c|c|c|c|}
\hline & 1 & 2 & 3 & 4 & 5 & 6 & 7 & 8 \\
\hline 1.Dimensión Afectiva & $493^{*+}$ & 1 & & & & & & \\
\hline $\begin{array}{l}\text { 2. Valoración de la asignatura y del } \\
\text { profesor de EF }\end{array}$ &, $247^{* * *}$ &, $131^{* 4 *}$ & 1 & & & & & \\
\hline 3. Dificultad de la EF &, $073^{*}$ &, $071^{*}$ & ,227 & 1 & & & & \\
\hline 4. Utilidad de la EF &,- 042 & $071^{*}$ &,$- 083^{*}$ & $275^{* *}$ & 1 & & & \\
\hline $\begin{array}{l}\text { 5. Empatía con el profesor y la } \\
\text { asignatura }\end{array}$ & $130^{* * *}$ & , $090^{* *}$ & , 38 &, $313^{* *}$ &, $094^{* *}$ & 1 & & \\
\hline $\begin{array}{l}\text { 6. Concordancia con la organización } \\
\text { de la asignatura }\end{array}$ & $164^{* *}$ & ,052 & , 325 &, $097^{* *}$ & $-1114^{*+}$ & $212^{* *}$ & 1 & \\
\hline 7. Preferencia por la EF y el Deporte &, $105^{* *}$ & $132^{* *}$ & $207^{+*}$ & $151^{* *}$ & $278^{* *}$ & $265^{* *}$ &, $070^{*}$ & 1 \\
\hline 8. La EF como Deporte & ,030 & ,056 & ,120" & $325^{+*}$ & $273^{* *}$ & $228^{* *+}$ &, $084^{*}$ & ,209" \\
\hline
\end{tabular}


serva en los datos de la siguiente tabla (tabla 1).

En el estudio comparativo entre la empatía y el sexo en adolescentes federados/as (tabla 2, figura 1), se detectan diferencias estadísticamente significativas (pd`0.05) en la dimensión cognitiva, propiciada por ser mayor en el sexo femenino ( $M=3.45)$ que en el masculino ( $M=3.38)$.

En el estudio comparativo entre la actitud hacia la EF y el sexo en adolescentes federados/as (tabla 3, figura 2), se detectan diferencias estadísticamente significativas (pd`0.05) en los ítems relacionados con la dificultad de la EF y la empatía con el profesor/a y la asignatura, en ambos casos los hombres puntúan más estos ítems que las mujeres.

Finalmente, en las correlaciones entre la empatía y actitud hacia la educación física (tabla 4), los resultados depararon correlación en casi todos los casos, en la dimensión cognitiva cuando esta se incrementa se genera un aumento hacia la valoración de la asignatura $\left(\mathrm{r}=0.247^{* *}\right)$, la dificultad de la misma $\left(\mathrm{r}=0.073^{*}\right)$, la empatía con el profesor y la asignatura $\left(\mathrm{r}=0.130^{* *}\right)$, concordancia con la organización de la asignatura $\left(\mathrm{r}=0.164^{* *}\right)$ y preferencia por la EF y el deporte $\left(\mathrm{r}=0.105^{* *}\right)$, en cuanto a la dimensión afectiva las correlaciones mostraban un aumento en esta, con un incremento en los ítems valoración de la asignatura $\left(\mathrm{r}=0.131^{* *}\right)$, la dificultad de la misma $\left(\mathrm{r}=0.071^{*}\right)$, utilidad de la EF $\left(\mathrm{r}=0.071^{*}\right)$, la empatía con el profesor y la asignatura $\left(\mathrm{r}=0.090^{* *}\right)$, y preferencia por la EF y el deporte ( $\mathrm{r}=0.132 * *)$.

\section{Discusión}

En este estudio se analiza la relación existente entre la empatía cognitiva y afectiva, y la actitud hacia la educación física en adolescentes federados/as andaluces. En primer lugar, el objetivo planteado en el análisis del estudio ha sido un descriptivo de la empatía, tanto cognitiva como afectiva, y de la actitud hacia la Educación Física. Los resultados nos muestran que la empatía cognitiva es mayor con respecto a la afectiva. Es posible que los procesos cognitivos y afectivos se den juntos o de forma separada. Así, y de acuerdo con los datos aportados por Kerem, Fishman y Josselson (2001), los procesos cognitivos son la antesala de los afectivos, y sólo es posible hablar de empatía si se dan los procesos cognitivos, con o sin su correlato afectivo. Esto demuestra que hay un mayor porcentaje de empatía cognitiva, ya que sin esta no se dará la empatía afectiva. Respecto a la actitud de los alumnos/as hacia la Educación Física, estudios como los de Moreno, Rodríguez y Gutiérrez (2003); Río y Giménez (2016), presentan como lo menos valorado el ítem de la concordancia con la Educación Física, siendo la asignatura y el profesor/a de Educación Física el más valorado, lo que es contrario a los datos obtenidos en esta investigación, donde lo más considerado es la concordancia con la Educación Física, y lo menos computado la utilidad de la Educación Física. El motivo puede ser que en la actualidad los alumnos buscan referentes, valorando más lo que se hace que lo que se dice.

En el estudio de la empatía según el género, los datos de este estudio muestran una diferencia en la empatía cognitiva entre hombres y mujeres, siendo mayor en las mujeres, al igual que en el estudio realizado por Navarro, Maluenda y Varas (2016) a alumnos/as universitarios, donde obtuvieron diferencias significativas en la empatía cognitiva y afectiva, siendo mayor la puntuación en las mujeres. Contrario a estos datos, Martínez-Otero (2011), realizó un estudio a universitarios que muestra que no existen diferencias significativas en la empatía cognitiva ni afectiva entre el sexo masculino y femenino. Esto puede ser debido a las diferencias de edad y al grado de madurez mayor en las mujeres en estas etapas.

Con respecto a la actitud que tienen los alumnos/as federados/as hacia la educación física, al igual que los datos que hemos obtenido, Moreno et al. (2003), recogen que se da mayor índice de empatía hacia el profesor y la asignatura por parte de los hombres. En contra, el estudio de Piéron, Castro y González (2006), muestra que existen diferencias de género en la realización de actividades con dificultad motriz, concretamente en la ejecución dentro de la educación física, como por ejemplo escalada técnica, siendo más practicada por los hombres que por las mujeres, lo que demuestra que los hombres tienen menos dificultad a la hora de realizar las actividades en Educación Física que la mujeres. Al igual que Ãàøè•-Ïàâèøè• y Äðàãàí, (2007) que muestran resultados positivos a nivel de actitud de educación física, obteniendo las mujeres, ligeramente, mejores puntuaciones a nivel de actitud y motivación que los hombres. Esto se debe a que el estudio se centró exclusivamente en deportes de alto rendimiento de manera individual. La causa de esto es por la mayor especialización desde edades tempranas en los hombres, a la hora de realizar deportes federados.

Por último, en cuanto a la relación de la empatía con la actitud hacia la Educación Física, diferentes estudios (Biddle et al., 1995; Cury, et al., 1996; Dorobantu y Biddle, 1997; Spray, 2002) determinan que la motivación y la actitud hacia la Educación Física están relacionadas a nivel de empatía tanto cognitiva como afectiva, lo que hace que si aumenta la motivación y la actitud hacia la Educación Física, aumente la empatía que a su vez hace que aumente la inteligencia emocional y con ello mejore sus relaciones sociales y su satisfacción con la vida. Otro estudio realizado por Altavilla, Furino, Di Palmo y Raiola (2015); Castillo, Torres, García y Buñuel, (2015), relaciona la empatía con la mejora del aprendizaje de los alumnos, siendo el verdadero aprendizaje significativo, aquel que muestra que las emociones no se oponen a la racionalidad. Los datos que hemos obtenidos nos muestran que a mayor empatía, sobre todo cognitiva, aumenta la actitud hacia la educación física, aumentando también su aprendizaje significativo.

Por último, en cuanto a la relación entre la empatía a nivel afectivo y la actitud hacia la Educación Física, Holt, Sehn, Spence, Newton y Ball, (2012) muestran una relación directa entre el aumento de empatía a nivel afectivo, tanto en el deporte escolar como en el deporte federado, con un aumento en todos los aspectos relacionados con la actitud hacia la educación física. Esto concuerda con los datos obtenidos en esta investigación, donde una mayor empatía se relaciona con un aumento en la totalidad de los componente de la actitud hacia la Educación Física.

Finalmente, es interesante conocer algunas de las principales limitaciones que se aprecian en esta investigación. La muestra que se ha utilizado debería de ampliarse para que sea más representativa de todos los y las escolares que realizan deporte federados en Andalucía, así como recoger más personas participantes federados en deportes denominados 
minoritarios.

En investigaciones futuras se debe seguir analizando sobre la relación que la empatía tiene directamente en la actitud del alumnado en la clase de Educación Física, y recoger los valores que las actividades extraescolares tanto federativas como no, tienen sobre la actitud que muestra el alumnado dentro del área de Educación Física. Además sería interesante relacionar estos datos con las conductas violentas, ya que en la actualidad, son un gran problema en los centros educativos.

\section{Conclusiones}

Las conclusiones más relevantes que se han obtenido del estudio son:

- El alumnado da un alto valor a la concordancia en las clases de Educación Física, siendo muy importante que el docente sea un modelo para sus alumnos, y que los contenidos tengan sentido para su vida diaria. Esto también queda reflejado en la obtención de los resultados que han sido menos valorados, donde el alumnado no ve utilidad en la Educación Física. Se valorará más si somos capaces de mostrar que la Educación Física no solo es competir, sino que tiene múltiples beneficios en el desarrollo tanto físico, psíquico y social.

- La empatía cognitiva presenta valores más elevado que la afectiva, mostrando que a esta edad los alumnos son capaces de conocer los sentimientos de los otros, pero son menos capaces de exteriorizarlos.

- Las mujeres muestran mayor empatía en la edad adolescente. Pero sin embargo, en relación a la actitud hacia la Educación Física, tiene una menor empatía hacia el profesor y la asignatura.

- Por último y de gran relevancia, cuanto mayor sea la empatía tanto a nivel cognitivo como afectivo, habrá un aumento en la actitud hacia la Educación Física. Un trabajo de Inteligencia Emocional en la etapa escolar, valorando la empatía, hará que el alumnado muestre una mejora en la actitud, con lo que se presupone una mejora en la motivación y en los resultados académicos.

\section{Bibliografía}

Altavilla, G., Furino, F., Di Palmo, M. y Raiola, G. (2015).Physicalskills, sport learning and socio-affectiveeducation, Sport Science, 8 (1), 44,46.

Anderson, C.A. y Bushman, B.J. (2002). Human aggression. AnnualReview of Psicología, 53, 27-51.

Aires, L., Silva, G., Alves, A.I., FilipaMedeiros, A., Nascimento, H., Magalhães, C., ... Mota, J. (2017). Longitudinal data from a school-basedintervention - The ACORDA project. Retos. Nuevas tendencias en Educación Física, Deporte y Recreación, 31, 207-211.

Beltrán, V. J.,Devís, J. y Peiró, C. (2012). Actividad física y sedentarismo en adolescentes de la comunidad valenciana. Revista Internacional de Medicina y Ciencias de la Actividad Física y Deporte, 12(45), 122-137.

Biddle, S., Cury, F., Goudas, M., Sarrazin, P., Famose, J. P. y Durand, M. (1995). Development of scaletomeasureperceivedphysicaleducationclassclimate: a crossnationalproject. British Journal of Educationalsychology, 65, 341-358.

Bouchard, C.,Shephard, R.J., Stephens, T., Sutton, J. y McPherson, B. A. (1990). Consensus of CurrentKnowledge. Exercise, Fitness and Health. Champaign. Human Kinetics.

Camacho-Miñano, M. J., García, E. F., Rico, E. R., y Ángel, J. B. (2013). La Educación Física escolar en la promoción de la actividad física orientada a la salud en la adolescencia: una revisión sistemática de programas de intervención. Revista complutense de educación, 24(1), 9-26.

Castillo, E. C., Torres, B. J. A., García, C. C. y Buñuel, P. S. L. (2015). Inteligencia emocional y motivación en educación física en secundaria. Retos: nuevas tendencias en educación física, deporte y recreación, (27), 8-13.

Chacón, R., Arufe, V., Espejo, T., Chacón, J., Zurita, F. y Castro, D. (2017). Práctica físico-deportiva, actividades de ocio y concepción sobre la Educación Física en escolares de A Coruña. Revista Retos: Nuevas tendencias en Educación Física, Deporte y Recreación, 32, 163-166.

Corbin, C. B., Pangrazi, R. P. y LeMasurier G. C. (2004). Physicalactivityforchildren: Currentpatterns and guidelines. President is Council of PhysicalFitness and Sport Researchdigest, 5(2), 1-8.

Cruz, J., Boixadós, M., Torregrosa, M. y Mimbrero, J. (1996). ¿Existe un deporte educativo?: Papel de las competiciones deportivas en el proceso de socialización del niño. Revista de Psicología del Deporte, 9, 111-132.

Cury, F., Biddle, S., Famose, J. P., Goudas, M., Sarrazin, P. y Durand, M. (1996). Personal and situationalfactorsinfluencingintrinsicinterest of adolescentgirls in schoolphysicaleducation: A structuralequationmodelinganalysis. EducationalPsychology, 16,305-315.

Dorobantu, M. y Biddle, S. (1997). Theinfluence of situational and individual goalsontheintrinsicmotivation of Romanianadolescentstowards PhysicalEducation. EuropeanYearbook of Sport Psychology, 1, 148-165.

Durán, J. (2013). Ética de la competición deportiva: Valores y contravalores del deporte competitivo. Materiales para la historia del deporte, 11, 89-115.

Eisenberg, N. y Fabes, R. A. (1998). Prosocialdevelopment. En W. Damon (Series Ed.), N. Eisenberg (Volumen Ed.). Handbookofchildpsychology: Social, Emotinal, and personalitydevelopment ( $5^{\text {a }}$ edición, vol. 3, pp. 701-778). Nueva York. Wiley.

Eisenberg, N., Guthrie, I. K., Murphy, B. C., Shepard, S. A., Cumberland, A. y Carlo, G. (1999). Consistency and Development of ProsocialDispositions: A longitudinal Study. ChildDevelopment, 70(6), 1360-1372.

Evans, M., Heriot, S.A. y Friedman, A.G. (2002). A behaviouralpattern of irritability, hostility and inhibitedempathy in children. ClinicalChildPsychology and Psychiatry, 7, 211-224.

Farrington, D. P., Jolliffe, D., Loeber, R., Stouthamer, M., y Kalb, L. M. (2001). Theconcentration of offenders in families, and familycriminality in theprediction of boys' delinquency. Journal of Adolescence, 24(5), 579-596.

García, M. (1997). Los españoles y el deporte (1980-1995) Un análisis sociológico. C.S.D. Valencia:Tirant lo blanch.

Goleman, D. (2011). WorkingWith Emotional Intelligence. New York. Bantam Books.

González, M., Manrique, J. C. y López, V. M. (2012). Valoración del primer curso de implantación de un programa municipal integral de deporte escolar. Retos: Nuevas Tendencias en Educación Física, Deporte y Recreación, 21, 14-18.

González, S. y Pastor, J. (2005). Revisión teórica y aplicación de un programa de enseñanza de valores a través del deporte educativo. En actas del I Congreso Internacional y XXIII Nacional de Educación Física. Universidad de Jaén.

Gorostiaga, A. y Balluerka, N. (2014). Evaluación de la empatía en el ámbito educativo y su relación con la inteligencia emocional. Revista De Educación, 364, 12-38.

Guillamón, A. R., García, E. y Pérez, J. J. (2018). Condición física y bienestar emocional en escolares de 7 a 12 años. Acta Colombiana de Psicología,21 (2), 282-300.

Gutiérrez, M., Tomás, J. M., y Calatayud, P. (2018). Determinantes de la práctica deportiva de los adolescentes en horario extraescolar. Revista Iberoamericana de Psicología del Ejercicio y el Deporte,13 (1), 91-100.

Hills, A., King, N. y Armstrong, T. (2007). TheContribution of PhysicalActivity and SedentaryBehaviourstotheGrowth and 
Development of

Children

and AdolescentsImplicationsforOverweight and Obesity. Sports $\mathrm{Me}$ dicine, 37(6), 533-545.

Hoffman, M.L. (2000). Empathy and moral development: Implicationsforcaring and justice.NewYork. Cambridge UniversityPress.

Holt, N. L., Sehn, Z. L., Spence, J. C., Newton, A. S., yBall, G. D. (2012). Physical education and sport programs at aninnercity school: exploring possibilities for positive youth development. Physical Education \& Sport Pedagogy, 17(1), 97-113.

Kane, J. E. (2015). Psychological aspects of Physical Edication and Sport .New York. Routledge.

Kaukiainen, A., Bjorkqvist, K., Lagerspetz, K., Osterman, K., Salmivalli, C., Rothberg, S. y Ahlbom, A. (1999). The relationship between social intelligence, empathy, and three types of aggression. Aggressive Behavior, 25, 81-89.

Kerem, E., Fishman, N. yJosselson, R. (2001). The experience of Empathy in every day relationships: Cognitive and affective elements. Journal of Social and Personal Relationships, 18 (5),709-729.

López, V. y Castejón, F. J. (2005). La enseñanza integrada técnicotáctica de los deportes en edad escolar. Explicación y bases de un modelo. Apuntes Educación Física y Deportes, 79, 40-48.

López, V. M., Pérez, D., Manrique, J. C. y Monjas, R. (2016). Los retos de la educación física en el siglo XXI. Retos, Nuevas tendencias en Educación Física, Deporte y Recreación, 29, 182187

López, P. D. M., Prieto, A., Samalot, A. y Madrona, P. G. (2016) Evaluación de una propuesta extraescolar de conductas apropiadas en educación física y deportiva. Retos: Nuevas Tendencias en Educación Física, Deporte y Recreación, 30, 36-42.

Martínez-Otero, V. (2011). La empatía en la educación: estudio de una muestra de alumnos universitarios. Revista Electrónica de Psicología Iztacala, 14(4), 174-190.

Manrique, J. C., López, V. M., Monjas, R., Barba, J. y Gea , J. M. (2011) Implantación de un proyecto de transformación socia en Segovia (España): Desarrollo de un programa de deporte escolar en toda la ciudad. Apuntes Educación Física y Deportes, 105 72-80.

Méndez-Giménez, A., Martínez de Ojeda Pérez, D. y Valverde-Pérez, J.J. (2016). Valoración del alumnado y profesorado del material convencional y auto- construido: estudio longitudinal de diseño cruzado en Educación Deportiva. Retos. Nuevas tendencias en Educación Física, Deporte y Recreación, 30, 20- 25.

Ministerio de Sanidad (2012). Las conductas relacionadas con la salud y el desarrollo de los adolescentes españoles: Resultados del estudio HBSC-2010 con chicos y chicas españoles de 11 a 18 años. Madrid: Ministerio de Sanidad, Servicios Sociales e Igualdad.

Moreno, J. A., Rodríguez, P. L., y Gutiérrez, M. (1996). Actitudes hacia la educación física: elaboración de un instrumento de medida. In Actas del III Congreso Nacional de Educación Física de Facultades de Educación y XIV de Escuelas Universitarias de Magisterio (pp. 507-516).

Moreno, J. A., Rodríguez, P. L. y Gutiérrez, M. (2003). Intereses y actitudes hacia la Educación Física. Revista Española de Educación Física, IX (2), 14-28.

Moreno, M. C., Muñoz, M. V., Pérez, P. J. y Sánchez, I. (2004). Los adolescentes españoles y su salud. Resumen del estudio ìHealth Behaviour in School aged Children. (HBSC-2002). Madrid: Ministerio de Salud y Consumo.

Muñoz, I. I. A., Ramos, J. M. G., y Benítez, M. M. Á. (2013). Deporte y competencias genéricas en la universidad: diseño y validación del «competest». Revista Complutense de Educación, 24(1), 141-163.

Navarro-Patón, R., Rodríguez, J. E. y Eirín, R. (2016). Análisis de la satisfacción de las necesidades psicológicas básicas, motivación y disfrute en Educación Física en Primaria. Sportis. Scientific Journal of School Sport, Physical Education and Psychomotricity, 2(3), 439-455.

Navarro, G., Maluenda, J. y Varas, M. (2016). Diferencias en empatía según sexo y área disciplinar en estudiantes universitarios chilenos de la provincia de Concepción, Chile. Educación, 25 (49), 63-82.

Nuviala, A., Ruiz, F., y García, E. (2003). Tiempo libre, ocio y acti- vidad física en los adolescentes. La influencia de los padres. Retos. Nuevas tendencias en Educación Física, Deporte y Recreación, 6, 13-20.

Ortiz, R. y Gómez, J. A. (2017). La actividad física, el entrenamiento continuo e intervalo: una solución para la salud. Revista Salud Uninorte, 33 (2), 252-258.

Piéron, M., Castro, M. J. y González, M. A. (2006). Actitudes y motivación en Educación Física escolar. Retos. Nuevas Tendencias en Educación Física. Deporte y Recreación, 10, 5-22.

Ramírez, W., Vinaccia, S., y Suárez, G. R. (2004). El impacto de la actividad física y el deporte sobre la salud, la cognición, la socialización y el rendimiento académico: una revisión teórica. Revista de estudios sociales, 18, 67-75.

Reloba, S., y Chirosa, L. y Reigal, R. (2015). Relación entre actividad física, procesos cognitivos y rendimiento académico de escolares: revisión de la literatura actual. Revista Andaluza de Medicina del Deporte, 9(4). 166-172.

Reloba, S., Reigal, R. E., Hernández, A., Martínez, E. J., Martín, I. y Chirosa, L. J. (2017). Efectos del ejercicio físico extracurricular vigoroso sobre la atención de escolares. Revista de Psicología del Deporte, 26, 29-36.

Reverter, J., Mayolas, M.C., y Adell, L. (2009). La competición deportiva como medio de enseñanza en los centros educativos de primaria. Retos: nuevas tendencias en educación física, deporte y recreación, 16, 5-8.

Río, J. F. y Giménez, A. M. (2016). El aprendizaje cooperativo: Modelo pedagógico para Educación Física. Retos: nuevas tendencias en educación física, deporte y recreación, (29), 201206.

Rojas, J. (1994). La violencia sin sentido. El País. 60, pp 61.

Romero, C. (2004). Argumentos sobre la formación inicial de los docentes en educación física. Profesorado, revista de currículum y formación del profesorado. Monográfico de Didácticas específicas, 8 (1), 1-20.

Ruiz, J. V., Ponce, A., Sanz, E. y Valdemoros, M. A. (2015). La educación en valores desde el deporte: investigación sobre la aplicación de un programa integral en deportes de equipo. Retos de la Actividad Física y el Deporte, 28,270-275.

Salovey, P. y Mayer, 1. D. (1990). Emotional intelligence. Imagination, Cognition. and Personality, 9. 185-211.

Serra, J. R., Zaragoza, J. y Generelo, E. (2014). Influences from «significant other» for physical activity practice in teenagers. Revista Internacional de Medicina y Ciencia de la Actividad Física y del Deporte. 14 (56), 735-753.

Spray, C. M. (2002). Motivationalc limate and perceived strategies to sustain pupils' discipline in physical education. European Physical Education review, 8, 5-20.

Stormont, M. (2002). Externalizing behavior problems in Young children: contributing factors and early intervention. Psychology in the School, 39, 127-138.

Strong, W., Malina, R., Blimkie, C., Daniels, S., Dishman, R., Gution, B.,... y Trudeau, F. (2005). Evidence based physical activity for school-age youth. Journal of Pediatrics, 146, 732-737.

Telama, R., Naul, R., Nupponen, H., Rychtecky, A. y Vuolle, P. (2002). Physical fitness, sporting life styles, and Olympic ideals: cross-cultural studies on youth sport in europe. Sport science studies. Schorndorf: Verlag Karl Hofmann.

Usán, P., Salavera, C., Merino, A. y Jarie, L. (2018). Satisfacción de necesidades psicológicas y orientaciones de meta en profesorado de Educación Física hacia sus alumnos. Retos. Nuevas tendencias en Educación Física, Deporte y Recreación, 33, 50-53.

Warburton, D.E., Nicol, C.W. y Bredin, S.S. (2006) Health benefits of physical activity: The evidence. Canadian Medical Association Journal,174,801-809.

Zurita, F., Fernández, R., Cepero, M., Zagalaz, M. L., Valverde, M. y Domínguez, R. (2009). The relationship between pain and physical activity in older adults that begin a program of physical activity. Journal of Human Sport and Exercise, 4(3), 248-297.

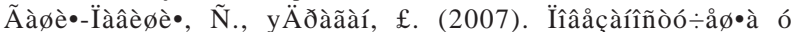

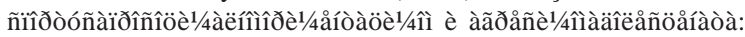
ðàçèèêåîîîिëó. ÇáîđíêêÉñnòèòóòçàïåäàâîêàèñòàcèâàœà, 39(2), 329-346. 